\title{
IgA Nephropathy, Celiac Disease and Dermatitis Herpetiformis: A Rare Triad
}

Arvind Gupta ${ }^{1}$ and Upma Narain ${ }^{2^{*}}$

${ }^{1}$ Department of Nephrology, Moti Lal Nehru Medical College, Allahabad, Uttar Pradesh, India

${ }^{2}$ Microbiologist \& Immunologist, Tejas Microdiagnostic, Allahabad, Uttar Pradesh, India

*Corresponding author: Upma Narain, Jawahar Lal Nehru Road, Allahabad, Uttar Predesh - 211002, India, Tel: +91-9415253337; E-mail: upmanarain@gmail.com Received Date: May 16, 2016; Accepted Date: June 30, 2016; Published Date: July 07, 2016

Copyright: (C) 2016 Gupta A, et al. This is an open-access article distributed under the terms of the Creative Commons Attribution License, which permits unrestricted use, distribution, and reproduction in any medium, provided the original author and source are credited.

\begin{abstract}
IgA nephropathy was found associated with celiac disease and dermatitis herpetiformis in a two years old male child at our center. This observed association widen the spectrum of the diseases that have been reported to coexist with IgA nephropathy.
\end{abstract}

Keywords: IgA nephropathy; Celiac disease; Dermatitis herpetiformis; Child; Gliadin antibody; IgA; Endomysial antibody

\section{Introduction}

IgA nephropathy occurs most often as a primary glomerulonephritis. However, its secondary form is commonly associated with liver disease [1]. Its etiology and pathogenesis are not very clear but the frequent occurrence of circulating immune complexes (IC) [2,3] and the presence of glomerular C3 deposits together with IgA suggest an immune complex mechanism. It is recently stated that abnormalities in the o-glycosylation of hinge region of IgA seems to be the best account for pathogenesis of IgA nephropathy [4]. Celiac disease and dermatitis herpetiformis (DH) are characterized by gluten-sensitive enteropathy, and they often occur in association with diseases of immunologic origin $[5,6]$ and, moreover, show circulating IgA ICs [7-9]. Thus we present a rare case of DH in a two year old male child previously diagnosed with celiac disease and IgA nephropathy at our center.

\section{Case Presentation}

Eight months back a two year old male child was presented with diarrhea, weight loss, bloating stomach pain, anorexia and anemia and was found difficulty in gaining weight. On investigations, hypoprotenemia $30 \mathrm{gm} / \mathrm{l}$, hypoalbunemia $15 \mathrm{gm} / \mathrm{L}$, proteinuria $1.5 \mathrm{gm}$ in $24 \mathrm{hrs}$ and dysmorphic RBCs were detected. Series of investigations were advised among which $\mathrm{S}$. creatnine and complement levels were normal ANA, dsDNA, MPO, PR3 and anti GBM antibodies were negative while IgA anti endomysial antibody, IgA anti-Gliadin antibody and tissue transglutaminase antibody were found positive. Renal Biopsy (Figure 1) was done and light microscopy revealed focal endocapillary proliferative glomerulonephritis with increased mesangial matrix and mesangial hypercellularity. Direct Immunofluoresence findings revealed IgG, C1Q, and fibrinogen did not show any fluoresence while $\operatorname{IgA}(3+$ mesangial granular) and IgM showed autofluoresence in tubules while C3 showed (1+ mesangial granular) autofluoresence in blood vessels. Thus diagnosis was made as Gluten sensitive enteropathy associated with IgA nephropathy.

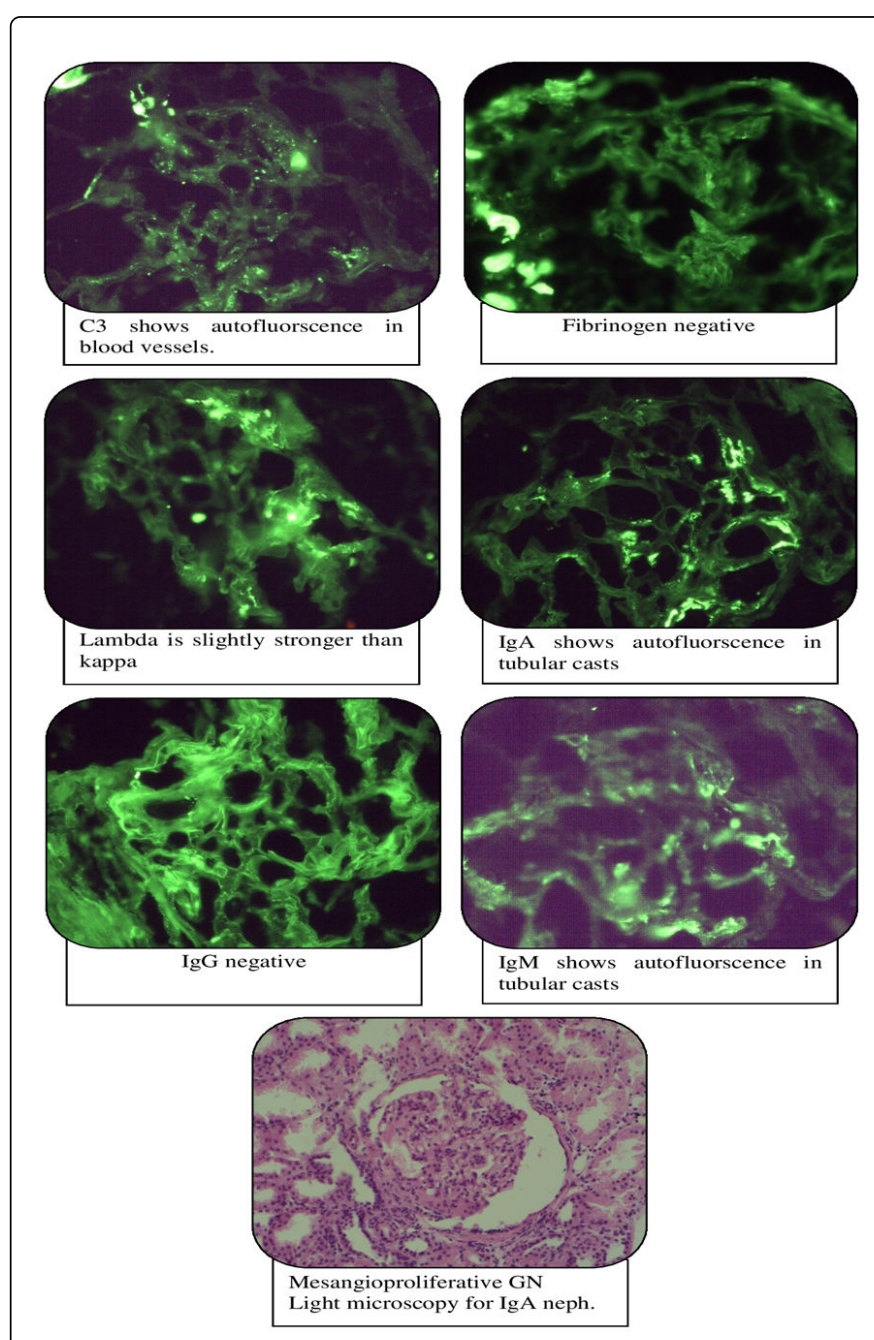

Figure 1: Direct immunoflurosence and light microscopy slides of renal biopsy. 
Gluten free diet started for celiac disease and $1.25 \mathrm{mg}$ daily Ramipril were started for nephritic syndrome. Child started recovering and was comfortable for 6 months and was on same treatment although, his proteinuria reduced was never resolved.

After six months of illness his proteinuria was increased up to 6.0 $\mathrm{gm}$ in 24 hours with mild deterioration in renal functions. Prednisolone $1 \mathrm{mg} / \mathrm{kg}$ started for renal relapse, and observed for two weeks. Proteinuria persisted to $5.0 \mathrm{gm}$ in 24 hours with hypo protenemia of $30 \mathrm{gm} / \mathrm{L}$. Myco phenolate sodium $360 \mathrm{mg}$ daily were added and prednesolone doses increased up to $1.5 \mathrm{mg} / \mathrm{kg} /$ day but there was no response in following two weeks. Then child (Figure 2) developed bilateral facial rash which was characterized by grouped excoriations, erythmatous and urticarial papular vesicles and were intensively pruritic lesions. Punch biopsy was done. Biopsy (Figure 3) of the lesions revealed a subepidermal blister with a predominantly neutrophilic infiltrate in the dermal papillary tips, although a mixed or even predominantly lymphocytic dermal infiltrate was also found. Direct immunofluorescence reveals granular deposition of IgA at the dermo-epidermal junction. Hence, diagnosis is made as DH. Dapsone at a dose of $1 \mathrm{mg} / \mathrm{kg}$ daily for a short period of time was started to control the local symptoms and to reduce the number and severity of skin injuries. Suddenly child developed septicemia, started deteriorating and unfortunately could not be saved.

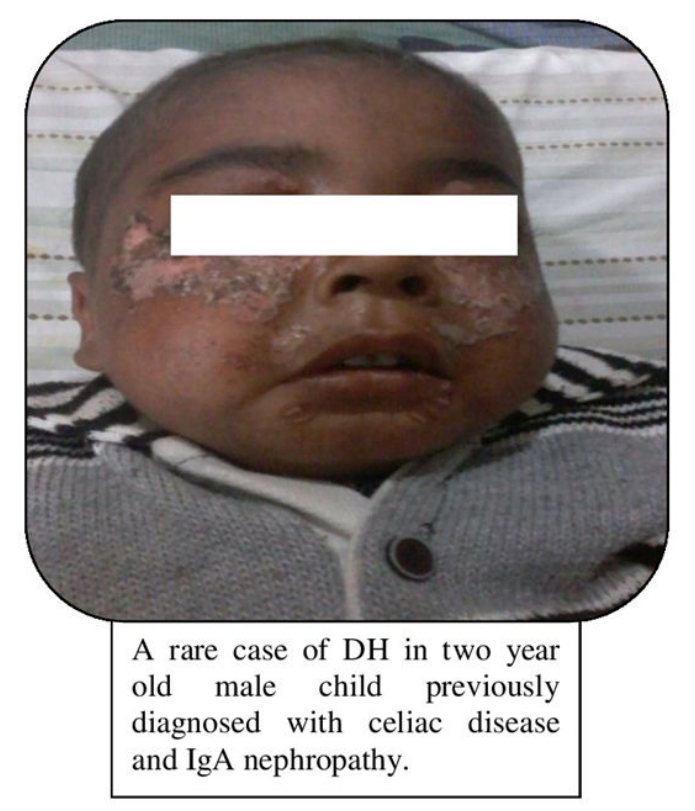

Figure 2: Bilateral facial rash which was characterized by grouped excoriations, erythmatous and urticarial papular vesicles

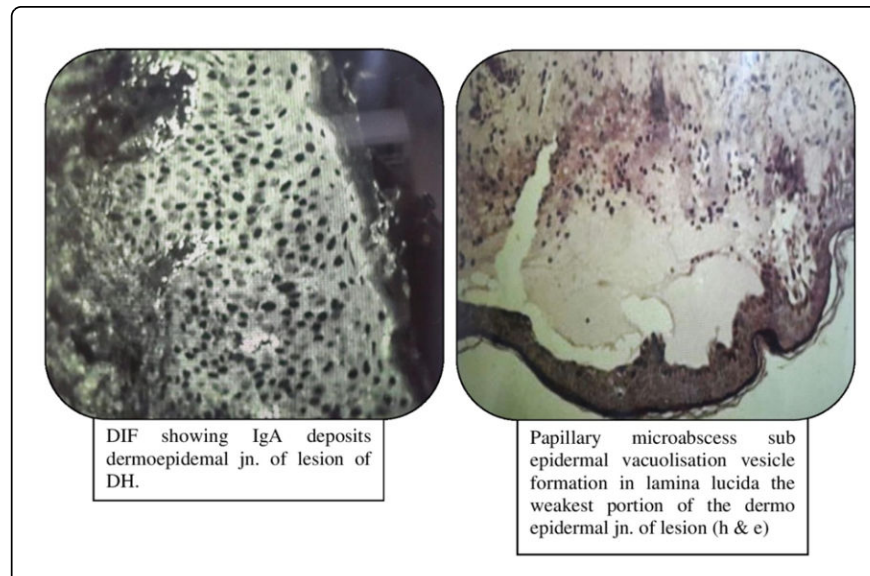

Figure 3: Direct Immunoflurosence and light microscopy slides of skin punch biopsy.

\section{Discussion}

The first report of mesangial IgA glomerulonephritis was reported by Berger and Hinglais, [10] they described this disease as primary glomerulonephritis. Several associations with other disease have since been documented, among which the Schonlein-Henoch syndrome, cirrhosis of the liver, ulcerative colitis and disease of colon are the best known. Coexistence of IgA nephropathy with small-cell bronchial carcinoma and cutaneous lymphoma has recently been reported. The present study adds to this list two closely related gluten-sensitive diseases, i.e. celiac disease and DH. It is well known that in primary IgA glomerulonephritis, dermal vessels often show IgA deposits, and it has been suggested that IgA deposition in the kidney may be just one manifestation of a systemic disease. IgA deposits were found in our patient in the papillary pattern or within the dermal vessel walls typical to $\mathrm{DH}[11]$.

Celiac disease (CD) is an autoimmune enteropathy triggered in genetically susceptible individuals by gluten ingestion. Individuals with this disease have permanent intolerance to the gliadin in fraction of wheat, barley and rye proteins [12-15]. It is the most common feeding intolerance in the occidental population and its estimated prevalence is $0.5 \%$ to $1 \%$ in the general population and 3 to 13 cases per 1000 children [13]. In its classical presentation the beginning of $\mathrm{CD}$ symptoms occurs generally in 6 to 24 months old children and is characterized by chronic diarrhea, abdominal pain, abdominal distension, insufficient growth, anorexia and vomiting [15]. This was very much distinct in our 2 year male child. However, many individuals can be asymptomatic and are diagnosed only after years of silent disease [13]. Atypical manifestation of CD includes extra intestinal changes as iron-deficient anaemia, osteoporosis, and cutaneous and neurological manifestations [15]. Our patient was anaemic too. The detection of high titres of antigliadin antibodies in the sera of IgAN patients has drawn attention of many authors to the possible relationship between IgAN and CD [16].

$\mathrm{DH}$ is a cutaneous manifestation of $\mathrm{CD}$. The association of $\mathrm{DH}$ with CD was initially described by Marks et al. 16 in 1966 [17]. The prevailing theory suggests that gluten sensitivity leads to the formation of IgA antibodies to gluten-transglutaminase complexes. These antibodies cross react with other transglutaminases, specifically to epidermal transglutaminase, which is highly homologous. Deposition 
of IgA-transglutaminase 3 complexes within the papillary dermis causes skin lesions of $\mathrm{DH}$. The tissue transglutaminase (tTG) was identified as the autoantigen prevalent in $\mathrm{CD}$, while in the $\mathrm{DH}$, the predominant autoantigen is the epidermal transglutaminase (eTG). Sardy et al proposed that the DH pathogenesis consists of immune response of low avidity for tTG, resulting initially in silent $C D$ that with continued exposure to gliadin, leads to the development of populations of antibodies with high affinity to eTG for cross-reactivity, which manifests as DH. Moreover, as in $\mathrm{CD}$, in which genetic predisposition is associated with the HLADQ2 (allelesDQA1 * 0501 and DQB $\left.1{ }^{\star} 020\right)$ and HLA-DQ8 (DQA1 ${ }^{\star} 03$ alleles and DQB1 ${ }^{\star} 0302$ ), DH presents similar genetic alterations $[18,19]$.

Childhood DH is rare, with an uncertain incidence and prevalence. Cases have been reported in children as young as 8 months, but most children receive the diagnosis between the ages of 2 and 7 years. Powell et al described a case with a predominance of urticarial lesions. Thus, childhood DH often is misdiagnosed as atopic dermatitis, papular urticaria, scabies, linear IgA dermatosis, or chronic urticaria. Recalcitrant cases of these diseases or patients who present with atypical findings of common diseases like atopic dermatitis should prompt the clinician to consider DH in the differential diagnosis [20].

Celiac disease and DH are often associated with other immunologic diseases, and there is a hypothesis that circulating ICs originating from the gut might be deposited not only in $\mathrm{DH}$ skin but also in various other organs causing the diseases. In IgAN, there is evidence of normal intestinal morphology but an increase in permeability has been described. Disordered mucosal permeability barrier can enhance antigen access to the immune system that further drives the production of pathogenic IgA and mesangial IgA depositions.

Recently, it was found that IgA deposited in DH skin is dimeric, which supports the concept that it originates from plasma cells in the gut. Like IgA glomerulonephritis, celiac disease and $\mathrm{DH}$ are diseases with frequent occurrence of circulating ICs. It has been shown that about $25 \%$ of patients with celiac disease and DH have IgA-class ICs in their serum. Favoring such a concept, circulating IgA antibodies were detected in our patient. Therefore it seems likely that in our patient, IgA glomerulonephritis could be related to their gluten-sensitive intestinal disease and $\mathrm{DH}$, via IC deposition in the kidneys [21].

\section{Conclusions}

Thus we reported a case of two year male child suffered with IgAN and celiac disease. Initially responded well to gluten free diet, but when his proteinuria relapsed and $\mathrm{DH}$ developed, his prognosis became worse. Unfortunately after giving so much effort we could not save the child with nosocomial infections.

\section{Declaration}

The authors declaring that the results presented in this paper have not been published previously in whole or part, except in abstract format.

\section{References}

1. Berger J, Yaneva H, Nabarra B (1977) Glomerular changes in patients with cirrhosis of the liver. Adv Nephrol Necker Hosp 7: 3-14.
2. Woodroffe AJ, Gormly AA, McKenzie PE, Wootton AM, Thompson AJ, et al. (1980) Immunologic studies in IgA nephropathy. Kidney Int 18: 366-374.

3. Mustonen J, Pasternack A, Helin H, Rilva A, Penttinen K, et al. (1981) Circulating immune complexes, the concentration of serum IgA and the distribution of HLA antigens in IgA nephropathy. Nephron 29: 170-175.

4. Kasper D, Fauci A, Hauser S, Longo D, Jameson JL, et al. (2015) Harrison's Principles of Internal Medicine (19th edn). McGraw-Hill Companies, New York.

5. Cooper BT, Holmes GK, Cooke WT (1978) Celiac disease and immunological disorders. Br Med J 1: 537-539.

6. Davies MG, Marks R, Nuki G (1978) Dermatitis herpetiformis--a skin manifestation of a generalized disturbance in immunity. Q J Med 47: 221-248.

7. Zone JJ, LaSalle BA, Provost TT (1980) Circulating immune complexes of IgA type in dermatitis herpetiformis. J Invest Dermatol 75: 152-155.

8. Hall RP, Lawley TJ, Heck JA, Katz SI (1980) IgA containing immune complexes in dermatitis herpetiformis, Henoch-Schonlein purpura, systemic lupus erythematosus and other diseases. Clin Exp Immunol 40: 431-437.

9. Hall RP, Strober W, Katz SI, Lawley TJ (1981) IgA-containing circulating immune complexes in gluten-sensitive enteropathy. Clin Exp Immunol 45: 234-239.

10. Berger J, Hinglais N (1968) Intercapillary deposits of IgA-IgG. J Urol Nephrol (Paris) 74: 694-695.

11. Helin H, Mustonen J, Reunala T, Pasternack A (1983) IgA nephropathy associated with celiac disease and dermatitis herpetiformis. Arch Pathol Lab Med 107: 324-327.

12. Cataldo F, Pitarresi N, Accomando S, Greco L, SIGENP, et al. (2004) Epidemiological and clinical features in immigrant children with coeliac disease: An Italian multicentre study. Dig Liver Dis 36: 722-729.

13. Imanzadeh F, Sayyari AA, Yaghoobi M, Akbari MR, Shafagh H, et al. (2005) Celiac disease in children with diarrhea is more frequent than previously suspected. J Pediatr Gastroenterol Nutr 40: 309-311.

14. McGough N, Cummings JH (2005) Celiac disease: A diverse clinical syndrome caused by intolerance of wheat, barley and rye. Proc Nutr Soc 64: 434-450.

15. Abenavoli L, Proietti I, Leggio L, Ferrulli A, Vonghia L, et al. (2006) Cutaneous manifestations in celiac disease. World J Gastroenterol 12: 843-852.

16. Sgnaolin V, Baldisserotto VR, Stein RT, Epifanio M (2013) Dermatitis herpetiformis as the only manifestation of celiac disease: Case report and literature review. Sci Med 23: 250-254.

17. Marks J, Shuster S, Watson AJ (1966) Small-bowel changes in dermatitis herpetiformis. Lancet 2: 1280-1282.

18. Karell K, Korponay-Szabo I, Szalai Z, Holopainen P, Mustalahti K, et al. (2002) Genetic dissection between coeliac disease and dermatitis herpetiformis in sib pairs. Ann Hum Genet 66: 387-392.

19. Mowat AM (2003) Celiac disease-a meeting point for genetics, immunology, and protein chemistry. Lancet 361: 1290-1292.

20. Templet JT, Welsh JP, Cusack CA (2007) Childhood dermatitis herpetiformis: A case report and review of the literature. Cutis 80: 473-476.

21. Unsworth DJ, Payne AW, Leonard JN, Fry L, Holborow EJ (1982) IgA in dermatitis-herpetiformis skin is dimeric. Lancet 1: 478-479. 